\title{
Optical coherence tomography complemented by hyperspectral imaging for the study of protective wood coatings
}

\author{
L.M. Dingemans ${ }^{\mathrm{a}, \mathrm{b}}$, V.M. Papadakis ${ }^{*}$, P. Liu ${ }^{\mathrm{b}}$, A.J.L. Adam ${ }^{\mathrm{a}}$, R.M. Groves ${ }^{\mathrm{b}}$ \\ ${ }^{a}$ Department of Imaging Science and Technology, Delft University of Technology \\ Lorentzweg 1, 2628 CJ Delft, The Netherlands; \\ ${ }^{\mathrm{b}}$ Aerospace Non-Destructive Testing Laboratory, Delft University of Technology \\ Kluyverweg 1, 2629 HS Delft, The Netherlands
}

\begin{abstract}
Optical coherence tomography (OCT) is a contactless and non-destructive testing (NDT) technique based on lowcoherence interferometry. It has recently become a popular NDT-tool for evaluating cultural heritage. In this study, protective coatings on wood and their penetration into the wood structure were measured with a customized infrared fiber optic OCT instrument. In order to enhance the understanding of the OCT measurements of coatings on real wooden samples, an optimization of the measuring and analyzing methodology was performed by developing an averaging approach and by post-processing the data. The collected information was complemented by data obtained with hyperspectral imaging to allow data from local OCT A-scans to be used in mapping the coating thicknesses over larger areas.
\end{abstract}

Keywords: optical coherence tomography, non-destructive testing, hyperspectral imaging, wood coatings, averaging, optimization, thickness mapping.

\section{INTRODUCTION}

The study of coatings on wood can provide information about damage to the coatings, the wood underneath, the penetration of the coating into the wood and the thickness of the coating. When selecting a non-destructive technique to obtain such data, both spatial and depth resolution will have to be considered as well as the light penetration. Using a shorter wavelength will give a better lateral resolution but will typically mean less penetration of light through the coating and the surface wood layers ${ }^{1}$. As a Non-Destructive Testing ( NDT) technique, Optical Coherence Tomography (OCT) has better spatial resolution than ultrasonic NDT and better depth penetration than microscopy. ${ }^{2}$ OCT has become a popular technology in the field of non-destructive testing with applications ranging from imaging damages in aerospace materials to examining museum objects ${ }^{3,4,5}$. At the same time, most OCT systems work in the near-infrared part of the light spectrum, meaning that for example white coatings with a thickness around $200 \mu \mathrm{m}$ cannot be imaged because the beam will not be able to penetrate into this coating deep enough to reach the wood-coating interface.

To study transparent and semi-transparent samples and to image their stratigraphy by reconstructing cross-sectional and volumetric images, OCT is a highly suitable technique. It will be shown that by using a $1.55 \mu \mathrm{m}$ low-coherent source, it is possible to study thin $(30-200 \mu \mathrm{m})$ transparent coatings on wood surfaces, their penetration into the wood structure and the drying process of these coatings. A reliable method has been constructed to post-process the obtained data, taking into account both surface irregularities as well as signal noise from the setup.

One of the main disadvantages of using OCT for the purpose of imaging coatings is the poor penetration of the light into thicker and less transparent coatings, along with the scanning time of OCT. A common OCT system can only scan an area a few millimeters by a few millimeters. To image a larger area, extra translation stages are needed, which requires extra design as well as tremendous scanning time. Hyperspectral imaging (HSI) is an optical technique that can quickly image large areas in small band-pass spectral bands. Although this technique does not specifically provide stratigraphic information, it is possible to map the thickness of a single surface layer ${ }^{7}$. It will be shown that it is possible to develop an

*v.papadakis@tudelft.nl; www.aerondt.tudelft.nl

Optics for Arts, Architecture, and Archaeology V, edited by Luca Pezzati, Piotr Targowski, Proc. of SPIE Vol. 9527, $952708 \cdot$ (c) 2015 SPIE · CCC code: 0277-786X/15/\$18 · doi: 10.1117/12.2184716 
iterative algorithm in order to first extract the spectra of the coating and of the wood underneath, then to determine the extinction coefficient using the thickness at a certain spot measured by the OCT and lastly to use this extinction coefficient to map the thickness of the coating in a larger area.

The aim of this paper is to evaluate OCT as a novel way of non-destructive imaging of protective wood coatings. In addition, information obtained using OCT will be used in combination with HSI in order to compensate the large scanning time of OCT. This is done by optimizing the signal and post-processing of the OCT data to retrieve sample parameters in a reliable way. These sample parameters are then used as input for an algorithm based on the HSI spectral data.

\section{MEASUREMENT DESCRIPTION}

\subsection{OCT principle \& experimental setup}

In OCT, the detection of the signal from a low-coherence source is based on a Michelson interferometer, which splits a source beam into a reference and a sample beam. The beam reflected from the sample will interfere with the reference beam, but only when the optical path lengths of the reference and sample beams are matched. The detector will then measure the intensity of the resulting interfering beams. Fig. 1 shows the two main OCT techniques, time-domain OCT (TDOCT) and Fourier-domain OCT (FDOCT). In TDOCT, a moving optical delay line (ODL) creates different optical path lengths in the reference arm which allows for scanning of the sample at different depths. FDOCT uses a stationary ODL and a spectrometer containing a grating and a linear detector, so that the amplitude of different wavelength components in the signal can be measured. Information on the depth of the reflections is encoded in the different wavelength components of the signal and can be recovered using an inverse Fourier transform. The great advantage of FDOCT is its fast data acquisition; however, a more elaborate system design is required in order to achieve an ideal signal-to-noise ratio (SNR). In both cases, a low-coherence source is used, which ensures that interference fringes are only observed when the path length difference between sample and reference beams is within the coherence length of the beam. The coherence length is thus a determining factor in the axial resolution of the OCT setup. In OCT, a point depth scan is also referred to as an A-scan.

In this study, coatings on wood surfaces and their penetration into wood structures were measured with a customized fiber optic OCT system. This prototype system was able to perform both TDOCT and FDOCT measurements, by using either an ODL (ODL-650-MC, OZ Optics, Ltd) or a NIR spectrometer (NIRQuest512, Ocean Optics Inc., US) respectively. The operational wavelength of the superluminescent diode (SLD, FESL-1550-20-BTF, Frankfurt Laser Company) source is centered at $1550 \mathrm{~nm}$, with a full width at half maximum (FWHM) of $60 \mathrm{~nm}$ and a coherence length of $34 \mu \mathrm{m}$ in air. The resulting spot size is $20 \mu \mathrm{m}$ and the axial resolution is $11 \mu \mathrm{m}$ inside the coating layer ( $\mathrm{n}=1.55)$.

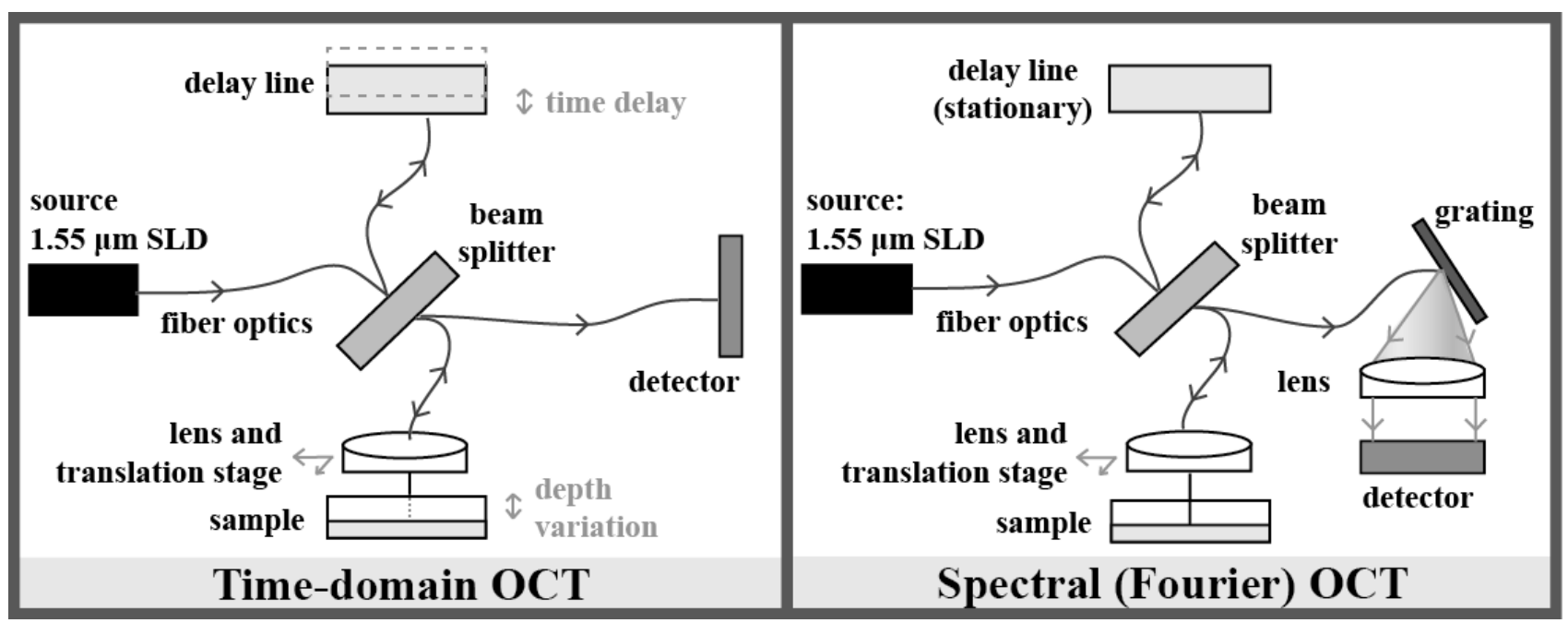

Figure 1. Two OCT techniques based on the Michelson interferometer. In TDOCT on the left, a moving delay line allows for scanning at different depths. In FDOCT on the right, a grating provides information about each wavelength component. 
An $x-y$ translation stage has been placed in the sample arm of the setup to allow for lateral scanning in two directions with a $28 \mathrm{~mm}$ range and a speed up to $4 \mathrm{~mm} / \mathrm{s}$. For further details on the setup, see Liu et al. ${ }^{8}$ and Wojtkowski ${ }^{9}$. After obtaining data via a LabVIEW ${ }^{\circledR}$ software program, a series of signal processing algorithms were applied. Bandpass filtering reduced excess signal noise and removed the DC component, after which an envelope detector was used to recover the depth dependent signal. Lastly, a median filter further smoothed the signal. The collected data were processed using Matlab ${ }^{\circledR}$. When plotting a 2D cross-section, the intensities were normalized relative to the maximum intensity in a cross-section by dividing all A-scans by this maximum intensity.

\subsection{Hyperspectral imaging principle \& experimental setup}

Hyperspectral Imaging (HSI) is a technique that combines digital imaging with reflectance spectroscopy and has a wide range of applications such as astronomy, remote sensing and medical sciences ${ }^{10}$. Roughly 25 years ago, HSI made an entrance in the field of non-destructive testing and in the field of cultural heritage $e^{11,12,13,14,15}$. In spectroscopy, the intensities of different wavelength components in a beam reflected, scattered or transmitted from or through a sample are measured. Because the absorption and scattering of light at a certain frequency can be related to a specific energy level and structure of the surface, a spectrum can provide very precise information about the surface composition of the sample. The source frequency for this type of measurement can be in the visible or the infrared range, with near infrared (NIR) light mostly used for obtaining information about the structure of a sample.

Combining spectrometry with imaging, a 3D space is provided called a spectral cube, with two spatial dimensions and one spectral dimension. In this way, the intensity of every wavelength at each pixel is mapped: $I(x, y, \lambda)$. This can be achieved either by acquiring images while scanning over the wavelength range or by collecting the whole spectrum for one point or line and scanning in the spatial dimension. The obtained spectral cube contains spectral information for each image pixel, as seen in Fig. 2.

The HSI setup used for this research, was an IMSPECTOR VIOE $\left(\right.$ Specim $\left.^{\odot}\right)$ spectral camera, operating in the 400-1000 $\mathrm{nm}$ wavelength range. The reflection spectra within this spectral range were collected on one spatial line on the sample of $30 \mathrm{~mm}$. Data were obtained using Specim DAQ solution software ${ }^{\odot}$, allowing for a full control of frame rate [Hz], exposure time [ms] and spectral and spatial binning. The system is equipped with an automated scanning platform in order to be able to scan the sample with an adjustable scanning and retrace speed [deg/s]. In order to enhance the information obtained about the UV part of the spectrum, a flattening filter $\left(\operatorname{Specim}^{\odot}\right)$ was used that suppressed the visible part of the spectrum. It is important to note that due to the geometry of the HSI setup, the incoming light has a 45-degree angle with respect to the sample while the detector is located parallel to the surface of the sample. This means that no specular reflections were recorded by the camera; instead, diffuse scattering caused by rough surfaces and scattering centers underneath the surface scattered the incoming beam in all directions, including partially in the direction of the sensor $^{11}$. As different materials scatter and absorb light in a different way, the spectrum collected in this way will contain information unique to this material. This geometry is also illustrated in Fig. 2.
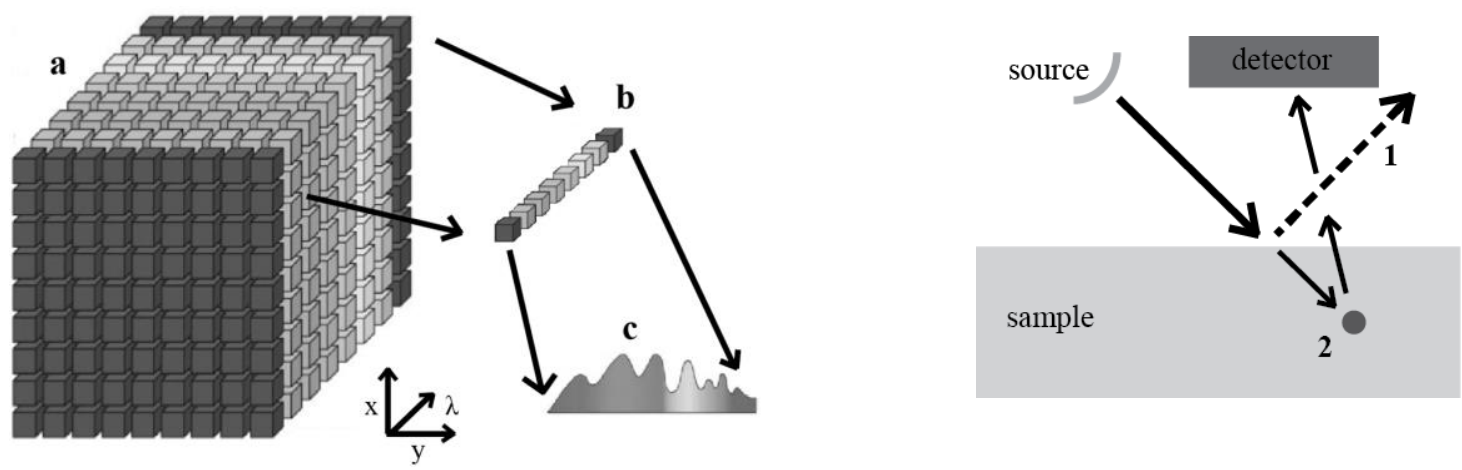

Figure 2. A hyperspectral imaging datacube (a), containing spectral information (b, c) per x-y spatial position. The geometry of the setup (right) is such that specular reflections (1) are not recorded, but diffuse scattering (2) is recorded. 


\subsection{Sample description}

The coating used in this research is a semi-transparent coating for protecting wood. The coating was applied to a spruce panel, selected as spruce is one of the most commonly used building materials in Europe. By applying the coatings in patches with varying thicknesses, an HSI spectral cube and OCT linescans could be collected containing information about the wood and various thicknesses of the coating. Freshly applied coatings have also been compared with coating that were applied on a spruce panel and then aged for roughly 2 years outside in The Netherlands. For reference measurements, the coating has also been deposited on a very flat silicon wafer as well as a glass plate.

\section{SIGNAL PROCESSING}

\subsection{Processing of TDOCT data}

One of the components of a TDOCT setup that can make it difficult to interpret the obtained data, is the ODL. This ODL should allow for a large enough axial scanning range, while it should also guarantee a stepwise movement which is much smaller than the axial resolution of the low-coherent light source. A non-linear or otherwise jittery ODL could significantly reduce the understanding of the OCT data, as illustrated in Fig. 3. For example, a non-linear motion of the ODL will make it difficult to compare two different point measurements, or A-scans, and derive the relative position of the reflection peaks from both measurements. When the ODL moves faster in the second measurement than in the first measurement, the reflection peaks appear to be occurring earlier as well, as if the reflections come from an interface closer to the source.

It is therefore important to have an understanding of features of the sample such as surface roughness as well as the nature of the noise in the data. These sample features can then be used to distinguish and also remove noisy data. This should be done in such a way that the yet unknown or uncertain sample features one wants to obtain information about, such coating thickness, are enhanced. In order to remove noise from the data, an averaging approach was introduced and will be explained below, after discussing how sample and noise features were studied.

\subsubsection{Sample and noise features}

The variety in the surface height can be used as an input parameter when post-processing the data and when trying to distinguish between jitter from the ODL in a TDOCT measurement and variations due to the surface of the sample. The variations in the surface roughness could for example be studied using a profilometer or a microscope. From literature, it is known that a single layer of protective lacquer on wood is usually fully absorbed by the wood and subsequent layers form surface coatings usually in the order of $30-150 \mu \mathrm{m}$ thickness ${ }^{16}$.

In order to map the behavior of the main source of noise in the TDOCT setup, the ODL, reference measurements were taken on a perfectly reflecting mirror (>95\% reflection) with a very small surface roughness in the order of $\lambda / 2$ to find the maximum error in determination of reflection peaks. For a large number of 50 point measurements at the same spot on a flat mirror, the noise was shown to be Gaussian, and can be dealt with accordingly as described below.

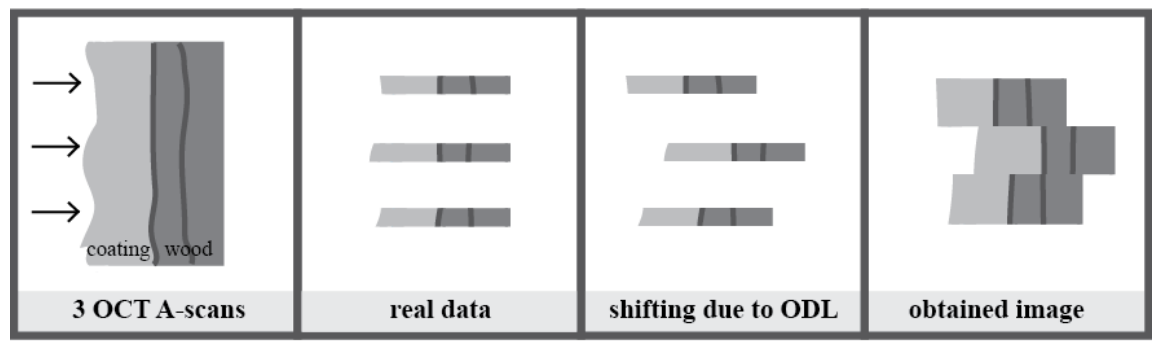

Figure 3. When the ODL movement is non-linear, the obtained OCT data are difficult to interpret 


\subsubsection{Correcting for noise by averaging}

For Gaussian noise, averaging the data over a number of point measurements will allow the position of the reflection peaks to converge to a certain value. In order to achieve such averaging, the same position was measured a number of times; Fig. 4 shows an averaging process over 5 A-scans, where the translation stage will move on to the next lateral position after measuring a position 5 times. In order to be able to determine the relative position of the signal, the first reflection peak is chosen as a reference point and was identified as the first peak above a certain threshold value.

One of the disadvantages of such an averaging approach is that even though the peak position of the A-scans at a spot can be determined more accurately, the averaged peaks will be broadened as well, as shown in Fig. 5. This is undesired because the width of the peak is a determining factor in the axial resolution: two closely spaced, broadened peaks are difficult to distinguish. By combining averaging with shifting of data, such broadening is circumvented. The process in Fig. 4 is used to determine the averaged peak position of the A-scan, after which the different A-scans at that lateral position are first all shifted such that their primary reflection peaks match the averaged primary reflection peak position. When the peaks are now added and divided, no broadening of the peaks will occur. This process is shown in Fig. 6 .

One of the disadvantages when applying such an averaging regime is that the duration of the measurement is highly increased because the measurement time is multiplied by the number of A-scans that is being averaged over at a single spatial point. Especially when large 2D lines or 3D areas are mapped, averaging the data over a large number of A-scans is not desirable. The number of A-scans should therefore be limited to a number that does significantly decrease the standard deviation in the primary peak position, while still being as small as possible. In order to find the optimum number of A-scans, the same data obtained to map the behavior of the ODL can be used; when averaging over different number of A-scans, the mean and standard deviation can be. In the Figures below, averaging over 5 A-scans is shown, while in many measurements an average over 4 was chosen to decrease the total duration of the measurement.

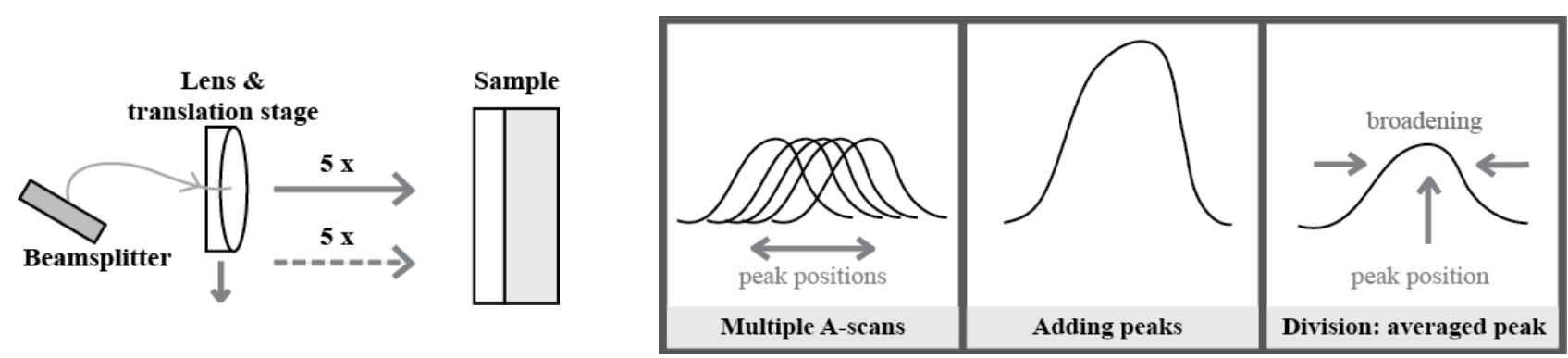

Figure 4. TDOCT averaging setup

Figure 5. TDOCT Averaging process

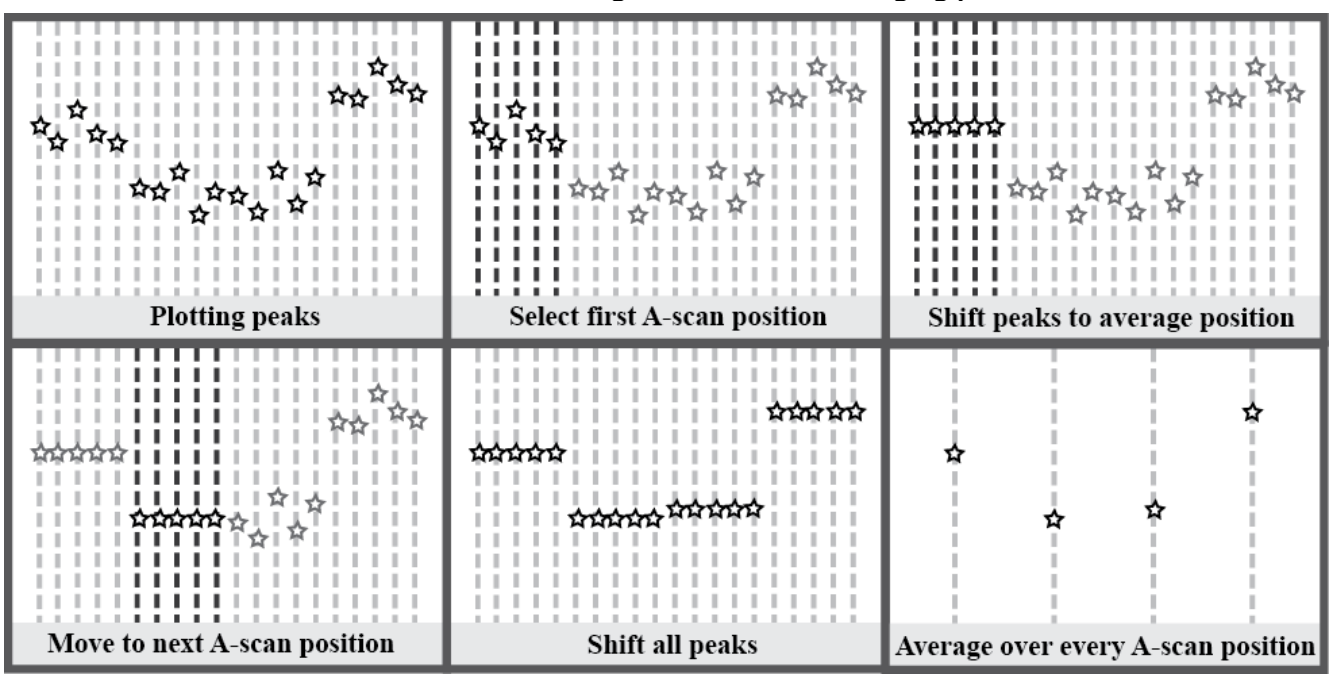

Figure 6. After obtaining 5 A-scans per lateral position, the average first peak-position is determined per 5 A-scans. After that, the 5 A-scans at that position are moved to the average position, before averaging. This process is repeated for all lateral positions. 
An error that can occur when applying such an averaging regime is that one of the A-scans could be an outlier due to a temporary large error in the ODL, therefore strongly affecting the average position to which the peaks are shifted. There are at least three possible solutions for this problem. The first solution would be to exclude peaks that are further away from the average peak position of the A-scans at a certain point than a threshold value. The disadvantage of this method is that because the average peak positions of these A-scans includes this faulty A-scan, the average is not very accurate and therefore may not exclude the faulty A-scan, or worse, exclude the correct A-scans instead. Another solution would be to exclude A-scans based on the approximate derivate of their position by comparing the position of one peak to its neighboring peak. However, when one averages over an $x$ number of A-scans, one can only obtain $x-1$ approximate derivatives in this way, making this method exclude a large part of the data. A last solution would be to determine the difference between a position and the average over all scans. This method is only possible when the surface is relatively flat, otherwise the threshold value will need to be set so large that locally, faulty A-scans will not be excluded. In the case of the relatively flat samples used in this research, the first solution was applied because no extreme outliers were present in most measurements.

\subsubsection{TDOCT measurement optimization without averaging}

When no averaging is used in obtaining data via OCT, optimization approaches can still be used to enhance the understanding of these data. Firstly, A-scans displaying reflection peaks at unlikely positions can still be identified and corrected for with a method similar to the one described in Section 3.1.2. The positions of the first and second reflection peaks can be located within an A-scan as the peaks above a certain threshold value. This threshold value should be related to the sample features as described in Section 3.1.1: any peaks lying further away from the average peak value than the maximum surface roughness of the sample, should be a result of ODL noise. Correcting of these peaks can be done by relocating the first reflection peak to the average position of the peaks spatially left and right from the faulty Ascan.

Secondly, the reflection peaks from these measurements can be artificially shifted in order to identify a typical relative position of the two interfaces of our samples (air-coating and coating-wood). First and second reflection peaks can be artificially aligned, creating a cross-sectional image where either the air-coating interface is assumed to be completely flat (by aligning the first reflection peaks) or the coating-wood interface is assumed to be flat (by aligning the second reflection peaks). Even though this would create an artificial image that does not take into account actual sample features such as surface roughness, these images can enhance the understanding of, for example, how the thickness of a coating varies spatially.

\subsection{Processing of HSI data}

Ideally, an algorithm should be developed that, when a sample of coated wood is scanned using HSI, can: extract the spectrum of the coating and the spectrum of the wood underneath in an automated way; determine the extinction coefficient of the coating using an OCT reference measurement for calibration; and - map the thickness of the coating throughout the sample (within the spatial area scanned with the HSI setup).

\subsubsection{Automated spectral un-mixing}

As one of the main applications of HSI is the field of planetary observations, a number of algorithms have been developed in order to be able to distinguish different substances. These algorithms are aimed at decomposing a dataset into spectrally pure constituent spectra of the materials present (endmembers) with corresponding fractions (abundances $)^{17}$. Recently developed algorithms try to extract endmembers from a dataset in an automated or unsupervised way, that is without providing reference spectra to the algorithm beforehand. The most frequently used algorithms are designed under the linear mixture model assumption, in which the reflectance $r_{i}$ at a specific wavelength $\lambda_{i}$ and pixel is denoted at a linear superposition of the reflectance of the different endmembers, multiplied by their abundance ${ }^{17}$ :

$$
r_{i}=\sum_{j=1}^{p} \rho_{i j} \alpha_{j}+n_{i}
$$


where $\rho_{i j}$ is the reflectance of endmember $j$ at wavelength $\lambda_{i}, \alpha_{i}$ is the abundance of that endmember and $p$ is the number of endmembers. Here, $n_{i}$ indicates an additive noise component. This model assumes minimal secondary and multiple scattering; some less commonly used models assume non-linear mixing and take into account multiple scattering. One of the most successful linear un-mixing algorithms is referred to as vertex component analysis (VCA), described by Nascimento et al. ${ }^{18}$

One of the limitations of the linear mixing model when applied to the un-mixing of hyperspectral data from measurements of coatings on wood, is that reflectance should not necessarily be seen as additive. The materials in the samples studied in this work are not an unordered mixture of different spectral components, but a neatly stratified twolayered system. It will however be shown that these algorithms can still give a valid approximation of the two components present in our coating-on-wood samples.

\subsubsection{Definition and determination of the extinction coefficient $k_{\lambda_{i}}$}

It is important to note that when the surface of the paint layer is assumed to be smooth, specular reflections from this surface will not be recorded as mentioned in section 2.2. However, the surface of the wood underneath has a higher roughness and will therefore scatter the incoming light in different directions, including partially the direction of the detector. Due to scattering and absorption in the coated layer, the intensity of the recorded light will be decreased by a wavelength-dependent term. In order to be able to model this scattering and absorption and relate it to the thickness of the coated layer, the absorption and scattering will be approximated using a formula that is in chemical texts usually referred to as the Lambert-Beer law ${ }^{19}$ :

$$
I_{\lambda_{i}}=I_{w, \lambda_{i}} e^{-2 k_{\lambda_{i}} \delta}
$$

where the measured intensity $I_{\lambda_{i}}$ at wavelength $\lambda_{i}$ is seen as a multiplication of the intensity of the light diffusely scattered from the wood at that specific wavelength, $I_{w, \lambda_{i}}$, and an exponential term taking into account the loss of intensity due to both scattering and absorption in the coating. The amount of absorption and scattering per wavelength band is dependent on the coating layer thickness $\delta$ and what will be referred to as the extinction coefficient $k_{\lambda_{i}}{ }^{13}$. This approximation holds under the assumption that almost all of the incoming light is transmitted through the first air-coating interface and only the first reflection from inside the material gives a significant contribution to the measured intensity. This scattering and absorption model is illustrated in Fig. 7.

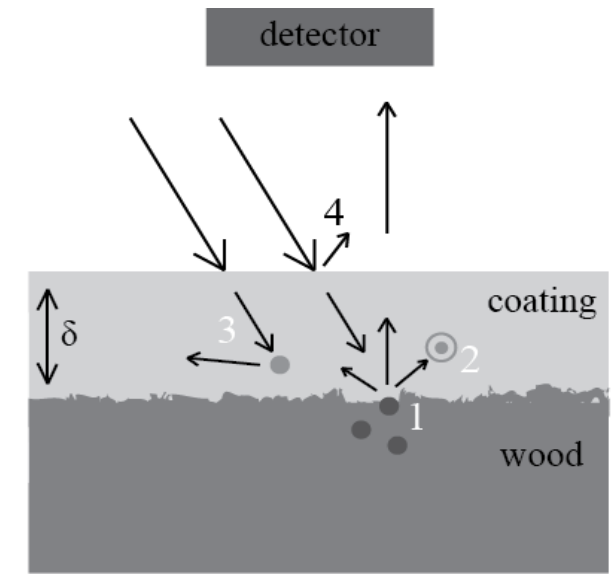

Figure 7. A two-layered sample with scattering from a rough wooden surface (1) and absorption (2), scattering in the coating layer (3) and specular reflection from the coating layer (4). 
The reflectance spectrum of the wood underneath the coating can either be determined using uncoated wooden reference samples or using the automated algorithm described above. The extinction coefficient can be determined with the help of an OCT measurement. OCT can determine the thickness of a coating layer at a specific point; as this thickness should remain constant over different wavelengths, reversing equation (2) can give the extinction coefficient:

$$
I_{\lambda_{i}}=\frac{1}{-2 \delta} \log \left(I_{\lambda_{i}} / I_{w, \lambda_{i}}\right)
$$

Another way to determine the extinction coefficient is by using a reference sample on a transparent material such as glass and measuring both the reflection and the transmission per wavelength band of a beam of light. It should be noted that for some wavelengths, the coating may become transparent and therefore it will not be possible to use the data from this part of the spectrum to determine the thickness of the coating. However, comparing calculated thicknesses values for different wavelength components within the absorbing part of the spectrum can validate the extinction coefficient, as the thickness should remain constant for different wavelengths.

\subsubsection{Thickness mapping}

When both the extinction coefficient $k_{\lambda_{i}}$ and the wood spectrum $I_{w, \lambda_{i}}$ are known for a specific wavelength, the thickness $\delta$ at a certain lateral position can be calculated from the measured intensity $I_{\lambda_{i}}$ of the HSI signal at that point. When this is done for the whole HSI spectral cube, a plot of the thickness of the coating is made. Due to an unavoidable error made in the calculation of the extinction coefficient, the thickness of the coating as determined in a thickness map may vary over different wavelengths. In order to correct for this, an iterative scheme has been envisioned, repeatedly adjusting the extinction coefficient based on the condition that the obtained thickness of the coating should remain constant (or within a certain set range). Such a scheme is visualized in Fig. 8 and can easily be implemented using Matlab ${ }^{\circledR}$.

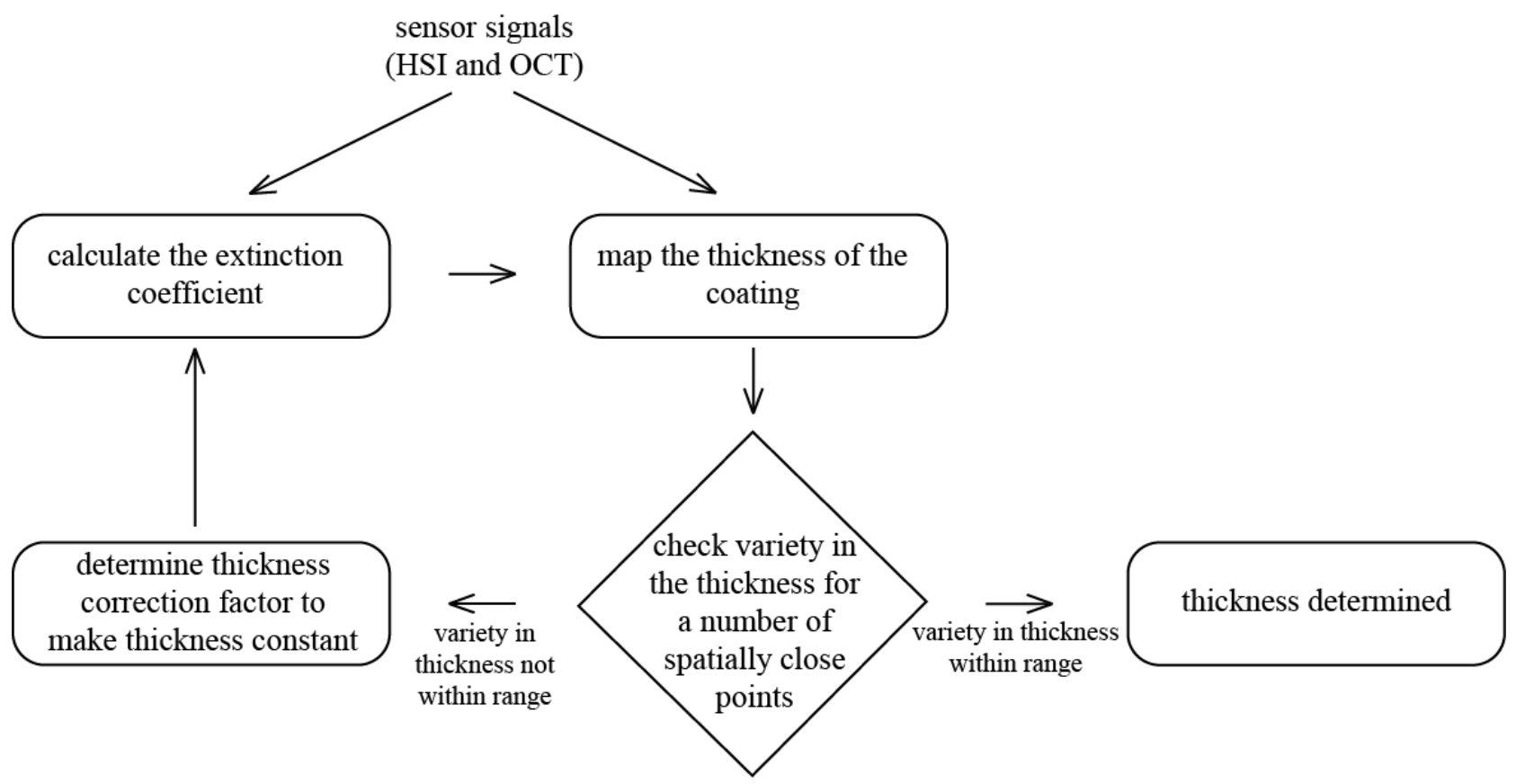

Figure 8. An iterative scheme for determining the thickness $\delta$ of a coating based on its extinction coefficient $k_{\lambda_{i}}$. 


\section{RESULTS}

\subsection{OCT signal optimization}

Before studying actual coatings on wood, an analysis of wood coatings with an optical thickness around $200 \mu \mathrm{m}$ on a silicon wafer were made as reference. These measurements show that both TDOCT and FDOCT are successful in imaging the coating, however, noise and artefacts distort the images. FDOCT has a much worse SNR at larger depths because the signal decays faster within the material, and for the purpose of imaging wood coatings, this technique is less suitable. The TDOCT reference measurements showed problems with the ODL, however, the averaging approach described in 3.1.2 proved to be successful on this flat reference sample, as shown in Fig. 9.

The same averaging approach was then applied to real coatings on wood, also showing promising results, as shown in Fig. 10. Here, the measurement of coated wood was averaged over 4 A-scans per lateral position. When averaging without shifting the peaks to an average position first, the final image shows both artefacts and broadening of the peaks.

\subsection{HSI thickness mapping}

An OCT linescan in 100 lateral positions averaged over 4 A-scans and with $150 \mu \mathrm{m}$ in between the lateral positions was performed in order to create a cross-sectional image of a large drop of coating on wood. The amount of coating applied was such that not all of the coating was absorbed into the wood, but a thick layer was left on top of the wood. This location was chosen so that the thickness of the drop could be determined in a more precise way by comparing the optical distance between the first and the second reflection peaks and was found to be approximately $320 \mu \mathrm{m}$. This thickness information was used as an input parameter for finding the extinction coefficient of the coating. Using formula (3), a reference wood spectrum and the spectrum collected with HSI at the thickest part of the drop of coating gave a value for the extinction coefficient for every wavelength band.
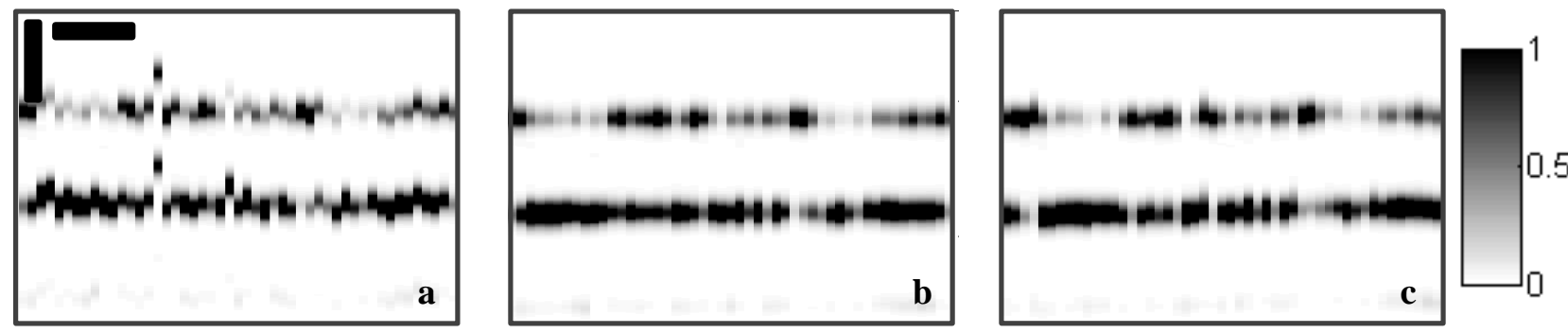

Figure 9. TDOCT measurements of a reference sample of coating on silicon wafer, line scan of 50 steps, with a step size of 10 $\mu \mathrm{m}$. (a): raw data, (b): these same data post-processed to align the primary peaks, and (c): scans averaged over 6 A-scans per lateral position. Vertical bar $200 \mu \mathrm{m}$, horizontal bar $100 \mu \mathrm{m}$.
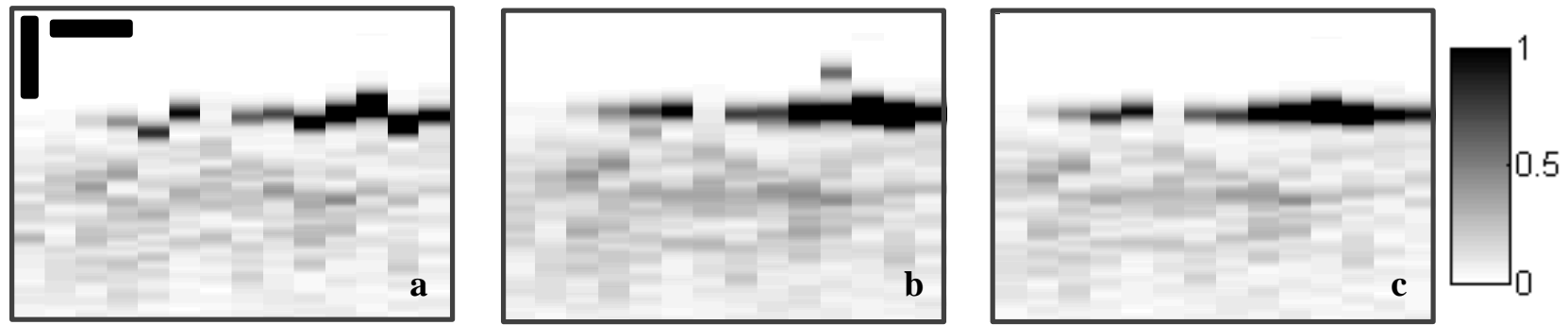

Figure 10. TDOCT measurements of coating on wood, line scan of 20 steps, $20 \mu \mathrm{m}$ in between the steps. (a): data without averaging, (b): averaging over 4 A-scans without shifting before averaging, (c): averaging after shifting A-scans to average position per lateral point. Vertical bar $200 \mu \mathrm{m}$, horizontal bar $60 \mu \mathrm{m}$. 
Using a different inversion of formula (2), this extinction coefficient was used to calculate the thickness of the coating layer in the whole HSI spectral cube. By plotting a line from this thickness plot, a thickness profile of the coating was imaged. The thickness of the drop and the thinner layer of coating next to the drop determined by this method are close to the actual thicknesses determined by the OCT linescan, as shown in Fig. 11.

The reference wood spectrum used in this measurement was determined by averaging over an area of the wood, whereas an automated algorithm could also have been used. The earlier mentioned VCA algorithm was applied to un-mix the HSI spectral cube to find the spectra of the wood and the coating. In particular finding the wood spectrum is relevant, because this can be used when inverting equation (2) to find the thickness of the coating. Fig. 12 shows the unmixed spectrum of the wood and the manually determined spectrum.


Figure 11. Combing OCT with HSI, a cross-sectional OCT image (a) can be used to determine the thickness $\delta$ of a coating, a parameter needed to determine the extinction coefficient $k_{\lambda_{i}}$ of the coating. This coefficient $k_{\lambda_{i}}$ is then used to map the thickness of the coating everywhere (b). A line from this data can generate very similar results as the OCT cross-section (c).

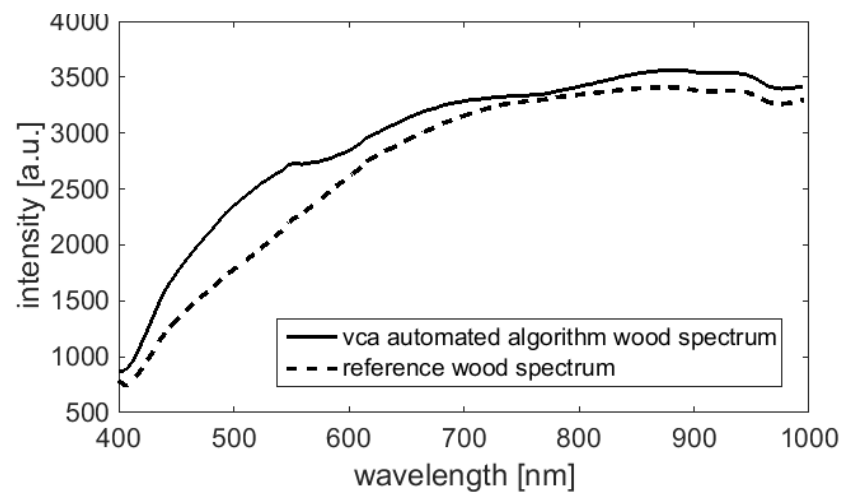

Figure 12. Spectra determined with an automated VCA algorithm and with manual averaging over a part of the wood surface. 


\section{DISCUSSION \& CONCLUSIONS}

This paper demonstrates a new application for OCT, a method of studying transparent and semi-transparent protective wood coatings. The results presented in this paper can be used as a preliminary study for further research in protective wood coatings. Collecting more OCT data will enhance the understanding of these data such that it will be possible to link OCT measurement to certain structural information about the wood or quality information about the coating.

In combination with HSI, OCT has shown to be a powerful calibration tool for mapping the thickness of a coating in an area. Further tests could show the conditions under which it is allowed to use the Lambert-Beer law to link absorption to coating thickness. The iterative scheme for mapping this thickness should still be enhanced so that the obtained thickness is always reliable. Enhancing the understanding of automated un-mixing algorithms will allow for improvements in the unmixed spectra, so that this step can be included in the iterative scheme and the mapping of the thickness can be done in an automated way.

\section{ACKNOWLEDGMENTS}

The authors kindly thank Akzo Nobel Coatings B.V. for the coatings with which the samples used in this research were prepared.

\section{REFERENCES}

[1] Fercher, A., Drexler, W., Hitzenberger, C. and Lasser, T., "Optical coherence tomography - principles and applications," Rep. Prog. Phys. 66, 239-303 (2003).

[2] Drexler, W. and Fujimoto, J., [Optical coherence tomography; technology and applications], Springer-Verlag, Berlin \& Heidelberg (2008).

[3] Liu, P., Groves, R.M. and Benedictus, R., "Quality assessment of aerospace materials with optical coherence tomography," Proc. SPIE 8430, 84300I-1-84300I-7 (2012).

[4] Targowski, P., Rouba, B., Wojkowski, M. and Kowalczyk, A., "The application of optical coherence tomography to the non-destructive examination of museum objects," Stud. Conservation 49, 107-114 (2004).

[5] Stifter, D., "Beyond biomedicine: a review of alternative applications and developments for optical coherence tomography," Appl. Phys. B 88, 337-357 (2007).

[6] Latour, G., Echard, J., Soulier, B., Emond, I., Variedelich, S. and Elias, M., "Structural and optical properties of wood and wood finished studied using optical coherence tomography: application to an $18^{\text {th }}$ century Italian violin," Appl. Opt. 48, 6485-6491 (2009).

[7] Papadakis, V., Loukaiti, A. and Pouli, P., "A spectral imaging methodology for determining on-line the optimum cleaning level of stonework," J. Cult. Herit. 11, 325-328 (2010).

[8] Liu, P., Groves, R.M. and Benedictus, R., "Non-destructive evaluation of delamination growth in glass fiber composites using optical coherence tomography," Proc. SPIE 9063, 90631M-1-90631M-9 (2014).

[9] Wojtkowski, M., "High-speed optical coherence tomography: basics and applications." Appl. Opt. 49, D30D61(2010).

[10] Papadakis, V.M., Karavellas, M.P., Tsilimbaris, M.K., Balas, C. and Pallikaris, I.G., "A hyper spectral imaging fundus camera for the detection and characterization of retinal lesions," Invest. Ophth. Vis. Sci. 4362-B331, 174 (2002).

[11] Kubik, M., [Physical techniques in the study of art, archaeology and cultural heritage], Elsevier BV, 5 (2007).

[12] Liang, H., "Advances in multispectral and hyperspectral imaging for archaeology and art conservation," Appl. Phys. A 106, 309-323 (2012).

[13] Papadakis, V.M., Orphanos, Y., Kogou, S., Melessanaki, K., Pouli, P. and Fotakis, C., "IRIS: a novel spectral imaging system for the analysis of cultural heritage objects," Proc. SPIE 8084, 80840W (2011).

[14] Balas, C., Papadakis, V.M., Papadakis, N., Papadakis, A., Vazgiouraki, E. and Themelis, G., "A novel hyperspectral imaging apparatus for the non-destructive analysis of objects of artistic and historic value," J. Cult. Herit. 4, 330-337 (2004).

[15] Melessanaki, K., Papadakis, V., Balas, C. and Anglos, D., "Laser induced breakdown spectroscopy (LIBS) and hyper-spectral imaging analysis of pigments on an illuminated manuscript," Spectrochim Acta B 57, 2337-2346 (2001). 
[16] Akzo Nobel Paint Belgium - Sikkens Bouwverven, "Technical information sheet," Sikkens, March 2015, $<$ http://docs.deco.akzonobel.com/webservices/dl/v1/Handlers/DocumentViewer.ashx?docid=5065c526-21afe111-8263-001f2954c954 > (20 May 2015). www.sikkens.be/nl/producten/detail/cetol_hls_plus

[17] Plaza, J., Hendrix, E., Garcia, I., Martin, G. and Plaza, A., "On endmember identification in hyperspectral imaged without pure pixels: a comparison of algorithms," Math Imaging Vis 42, 163-175 (2011).

[18] Nascimento, J., Bioucas Dias, J., "Vertex Component Analysis: a fast algorithm to unmix hyperspectral data," IEEE Trans. Geosci. Remote Sens. 43 (4), 898-910 (2005).

[19] Bohren, C., Clothiaux, E., [Fundamentals of atmospheric radiation; An introduction with 400 problems], Wiley, Weinheim (2006). 